\title{
DEMONSTRATION OF PREAMBLE LESS OPTICAL PACKET CLOCK AND DATA RECOVERY WITH OPTICAL PACKET SWITCHING
}

\author{
Naoya Wada', Hatsushi Iiduka ${ }^{2}$, and Fumito Kubota ${ }^{1}$ \\ ${ }^{\prime}$ National Institute of Information and Communications Technology (NICT), 4-2-1, Nukui- \\ Kita, Koganei, Tokyo 184-8795, Japan.wada@nict.go.jp \\ ${ }^{2}$ NTT Electronics Co., 1841-1, Tsuruma, Machida, Tokyo 194-0004, Japan. \\ ohtsuka@atsul.nel.co.jp
}

\begin{abstract}
A novel preamble free optical packet 3R receiver is proposed. Optical packets stream composed of $10 \mathrm{Gbit} / \mathrm{s}$ preamble-less payload data and arbitrary intervals is generated and received with instantaneous clock and payload data recovery in less than a bit period $(<100 \mathrm{ps})$. Preamble-less optical packet clock and data recovery with two hop 40Gbit/s optical packet switching based on all-optical code label processing is experimentally demonstrated.
\end{abstract}

\section{INTRODUCTION}

The next generation of optical network will need high scalability and fine granularity as well as large network capacity. Wavelength division multiplexing (WDM) technology has a huge capacity for data transmissions. However, the granularity of a WDM light-path network is coarse. Networks based on optical packet switching (OPS) can provide high scalability, fine granularity and ultrahighspeed hopping. Despite their lack of maturity, many OPS systems have been developed because of their obvious merits [1-5].

Optical packet 3R receiver will be a very important component in a future OPS networks. Some optical burst-mode $3 R$ receivers have been reported [6-8]. 
However, these burst-mode receivers require preamble pattern and/or training bits for stable clock and payload data recovery. Therefore, time for clock and data recovery of these burst-mode receivers is nanoseconds order. Such overhead time due to preamble deteriorates the network performance.

In this report, a novel preamble free optical packet $3 \mathrm{R}$ receiver is proposed. $10 \mathrm{Gbit} / \mathrm{s}$ optical packets streams with 1000 to 4000 bits-long, preamble-less, payload data and arbitrary intervals from nanosecond to microseconds are tested. The packets stream is asynchronously received by optical packet $3 \mathrm{R}$-receiver. Instantaneous clock and payload data recovery in less than a bit period $(<100 \mathrm{ps})$ is experimentally demonstrated. In addition, preamble-less optical packet clock and data recovery with two hop $40 \mathrm{Gbit} / \mathrm{s}$ optical packet switching based on all-optical code label processing and optical time-domain multiplexing and de-multiplexing technologies is experimentally demonstrated.

\section{OPTICAL PACKET 3R RECEIVER}

The block diagram of proposed optical packet 3R receiver is shown in Fig.1. Optical packet 3R-receiver consists of $\mathrm{O} / \mathrm{E}$ converter and clock and data recovery (CDR) parts. Specialy tuned uni-traveling-carrier photo-detector (UTC-PD) is introduced as an $\mathrm{O} / \mathrm{E}$ converter. CDR consists of phase shifter (T/2), X-OR, D-FF, and low jitter gated VCO. The block diagram of proposed low jitter gated VCO is shown in Fig.2.

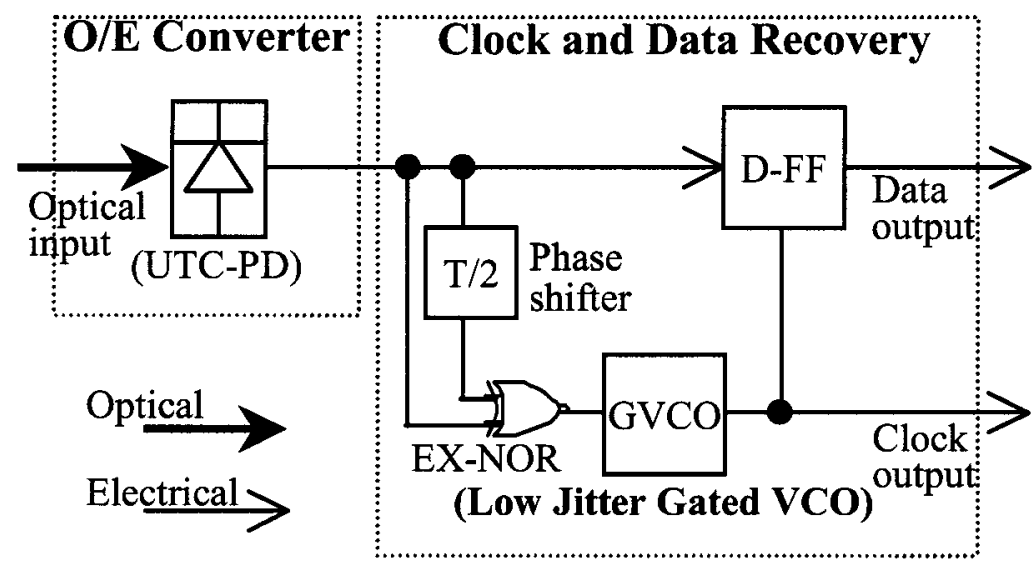

Figure 1. Block diagram of optical packet 3R receiver 


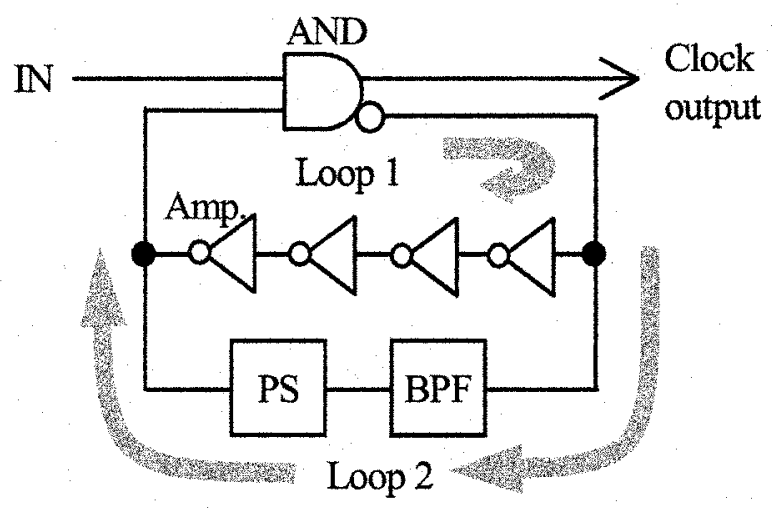

Figure 2. Block diagram of low jitter gated VCO

PS: Phase shifter

The low jitter gated VCO has the double phase lock loop structure. Loop 1 is for start up operation at around $10 \mathrm{GHz}$. Loop 2 is for very low jitter operation at $9.95328 \mathrm{GHz}$ after loop 1 .

\section{EXPERIMENTAL DEMONSTRATION I: PREAMBLE LESS OPTICAL PACKET CLOCK AND DATA RECOVERY}

Figure 3 represents an experimental set-up of an instantaneous clock and payload data recovery and bit error ratio (BER) measurement. The set-up consists of a $10 \mathrm{GHz}$ mode locked laser diode (MLLD) as a light source, $\mathrm{LiNbO}_{3}$ intensity modulator (LN-IM), pulse pattern generator (PPG), optical attenuator, proposed optical packet $3 \mathrm{R}$ receiver, sampling oscilloscope and error detector. Optical amplifiers (EDFA) were employed to compensate insertion losses of the components in combination with optical band-pass filters (BPF) to attenuate the amplified spontaneous emission. The MLLD generates 1.5ps pulses with $9.95328 \mathrm{GHz}$ repetition rate at central wavelength of $1550 \mathrm{~nm}$. This pulse train is modulated by a LN-IM to generate optical packets streams with 1000 to 4000 bitslong, preamble-less, return-to-zero (RZ) payload data and arbitrary intervals from nanosecond to microseconds. The generated packets streams are input to the optical packet $3 R$ receiver via optical fiber and attenuator. In the $3 R$ receiver, Instantaneously, clock and payload data are recovered from asynchronously received preamble-less optical packet data stream. The recovery time is less than a bit period $(<100 \mathrm{ps})$. The waveform and BER of recovered payload data are measured by sampling oscilloscope and error detector, respectively. 


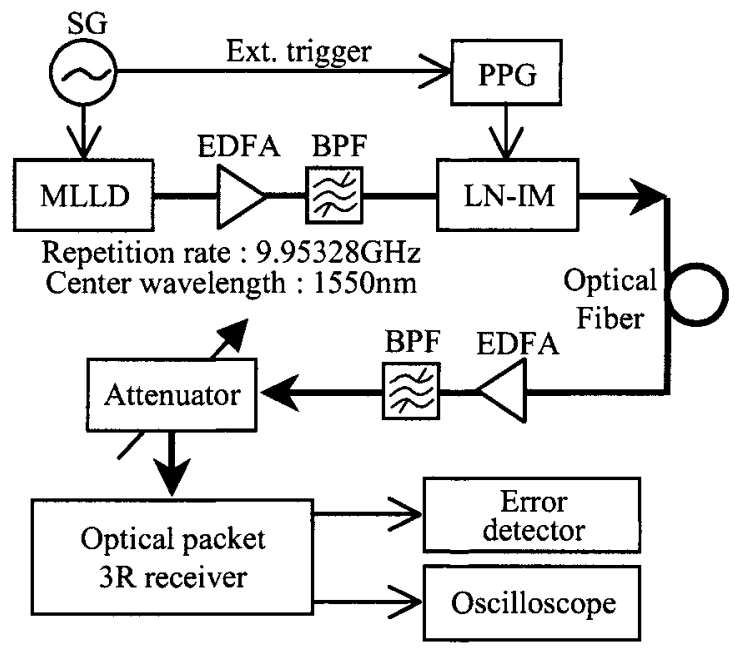

Figure 3. Experimental set-up of preamble less optical packet clock and data recovery
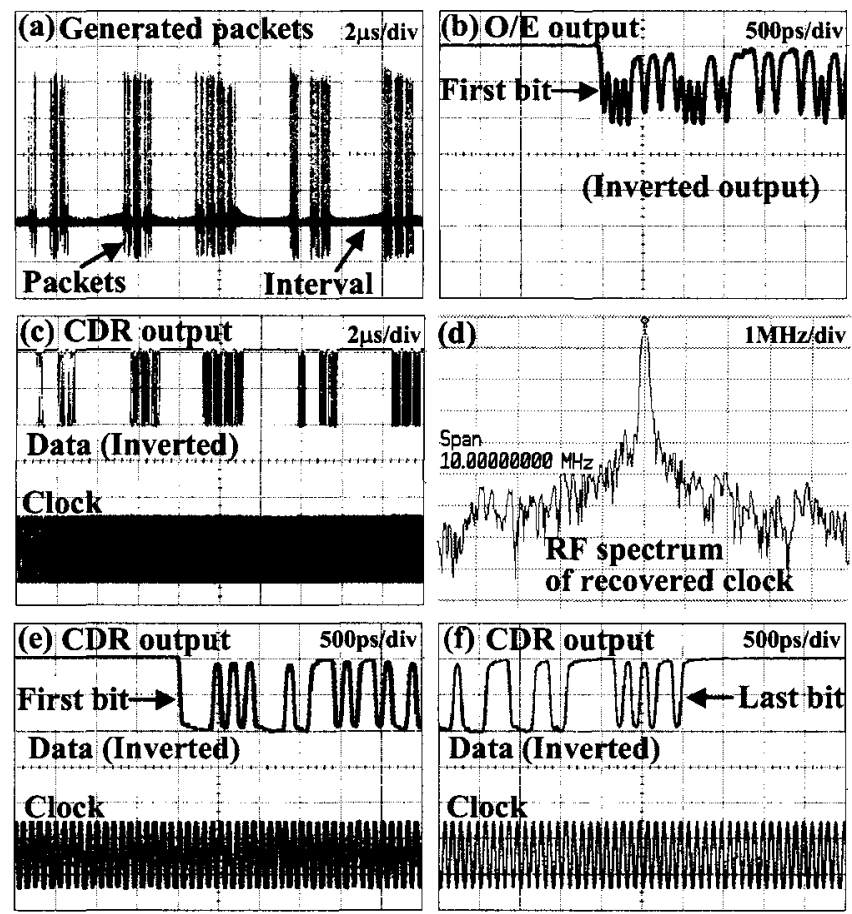

Figure 4. Measured waveforms of experimental demonstration I 
Figure 4 (a) is the waveform of a generated random optical packet stream. In the $\mathrm{O} / \mathrm{E}$ part of $3 \mathrm{R}$ receiver, input optical packets are converted to electronic signal via UTC-PD with $40 \mathrm{GHz}$ bandwidth. Inverted waveform of the $\mathrm{O} / \mathrm{E}$ output is shown in Fig. 4(b). Figure 4 (c) represents Inverted waveforms of CDR outputs. Recovered data and clock are represented in upper and lower parts, respectively. Figure 4 (d) is RF spectrum of recovered clock by CDR. This RF spectrum shows the stable clock recovery of the optical packet $3 R$ receiver. Figures 4 (e) and (f) are magnification of Fig. 4 (c). Figure 4 (e) is the beginning of the recovered data and clock. Figure 4 (f) shows the ending of the recovered data and clock. These results represent the instantaneous clock and payload data recovery in less than a bit period.

Figure 5 represents measured BERs in the optical packet receiving experiment. Figure 5 (a) shows measured BER and eye diagram of the recovered payload data from a random packet stream represented in Fig. 4(c). This stream consists of 4000 bits-long, preamble-less, payload data and arbitrary intervals from nanosecond to microseconds. Figure 5 (b) shows measured BERs of three different regular packet streams consist of 1000,2000 , and 4000 bits-long, preamble-less, payloads, respectively. In each case, the stream has about $100 \mathrm{~ns}$ intervals between packets. These results guarantee instantaneous and stable clock and payload recovery for packets data.

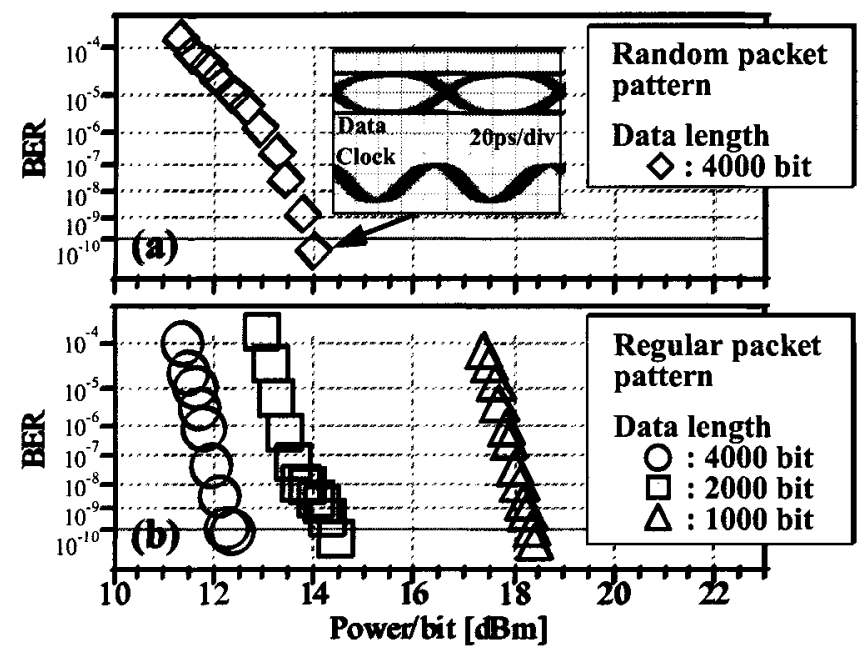

Figure 5. Measured BERs and eye diagram of experimental demonstration I 


\section{EXPERIMENTAL DEMONSTRATION II: PREAMBLE LESS OPTICAL PACKET RECEIVING WITH TWO HOP OPTICAL PACKET SWITCHING}

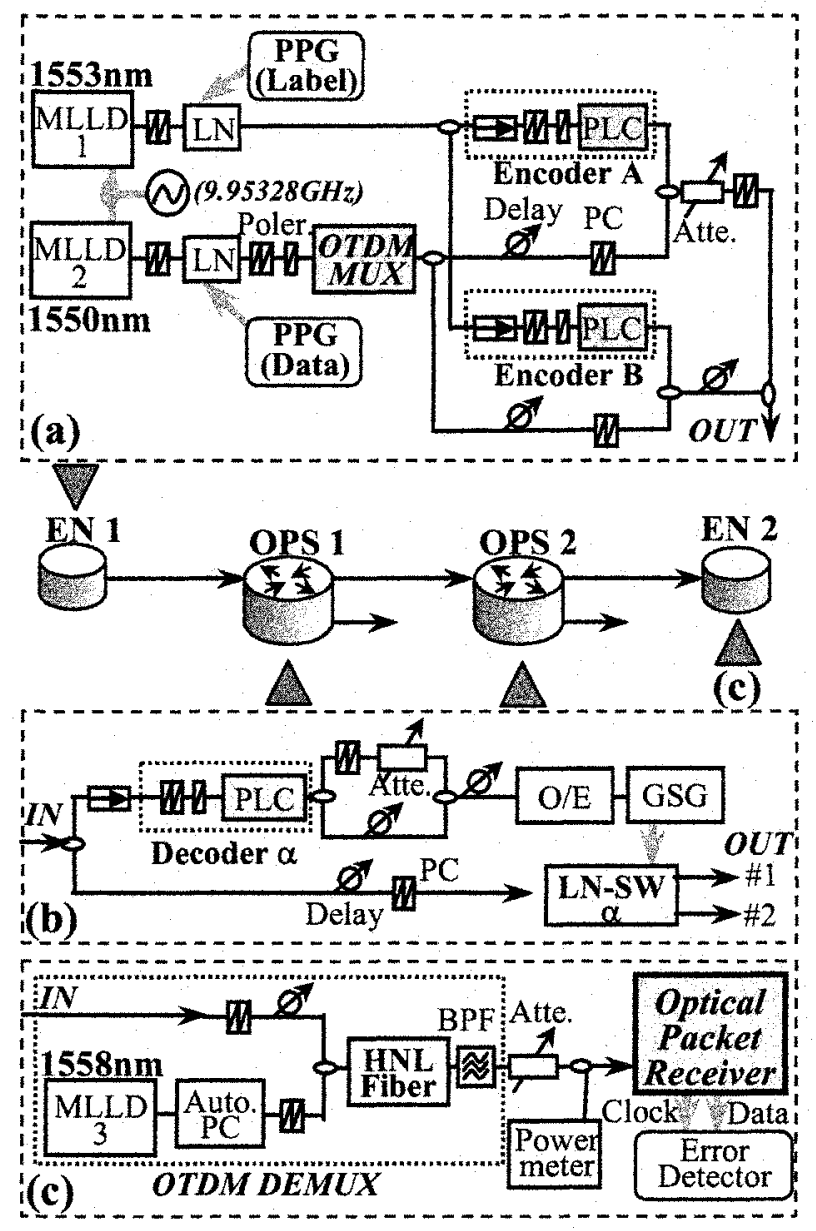

Figure 6. Experimental set-up demonstration II

Figure 6 represents experimental set-up of preamble less optical packet receiving with two hop optical packet switching. Fig. 6(a) and (c) are setup of source node (EN1) and destination node (EN2), respectively. Fig. 6(b) represents OPS nodes 1 and 2. 40Gbit/s packets with optical code label are generated in EN1 and switched two times by OPS 1 and 2. Finally, switched packets are received by EN2. In the experiment, we use packets with label $A$ and $B$. EN1 consists of $10 \mathrm{GHz}$ mode locked laser diode (MLLD), $\mathrm{LiNbO}_{3}$ intensity modulators (IM), 
Planer lightwave circuit (PLC) based encoders to generate 8-chip, 200Gchip/s optical bipolar label, optical delay, and OTDM multiplexer (MUX). Second IM generates $10 \mathrm{Gbit} / \mathrm{s}, 4000 \mathrm{bits}$ random packet data. This $10 \mathrm{Gbit} / \mathrm{s}$ data converted to 40Gbit/s, 16000bits data by an OTDM-MUX. Generated optical label and 40Gbit/s packet data are combined to form an optical packet. OPS 1 and 2 consist of label processor and optical switch, which correspond upper and lower arm of Fig. 6(b), respectively. Label processor consists of PLC decoders as optical correlator, photo detectors (PD), gate signal generator (GSG), BPF, and optical delay. Table lookup of the packet label is performed in a parallel manner without $\mathrm{O} / \mathrm{E}$ and $\mathrm{E} / \mathrm{O}$ conversions [2]. Label processor control optical switches via GSG to rout packets to their designated output port. $\mathrm{A} 1 \times 2 \mathrm{LiNbO}_{3}$ optical switch is used in the experiment. Finally, switched packets input EN 3. In EN3, 40Gbit/s data is demultiplexed to $10 \mathrm{Gbit} / \mathrm{s}$ data by using local MLLD and high nonlinear fiber (HNL Fiber) and received by the optical packet receiver. Optical amplifiers (EDFA) were employed to compensate insertion losses of the components in combination with $5 \mathrm{~nm}$ bandwidth optical band-pass filters (BPF) to attenuate the amplified spontaneous emission.
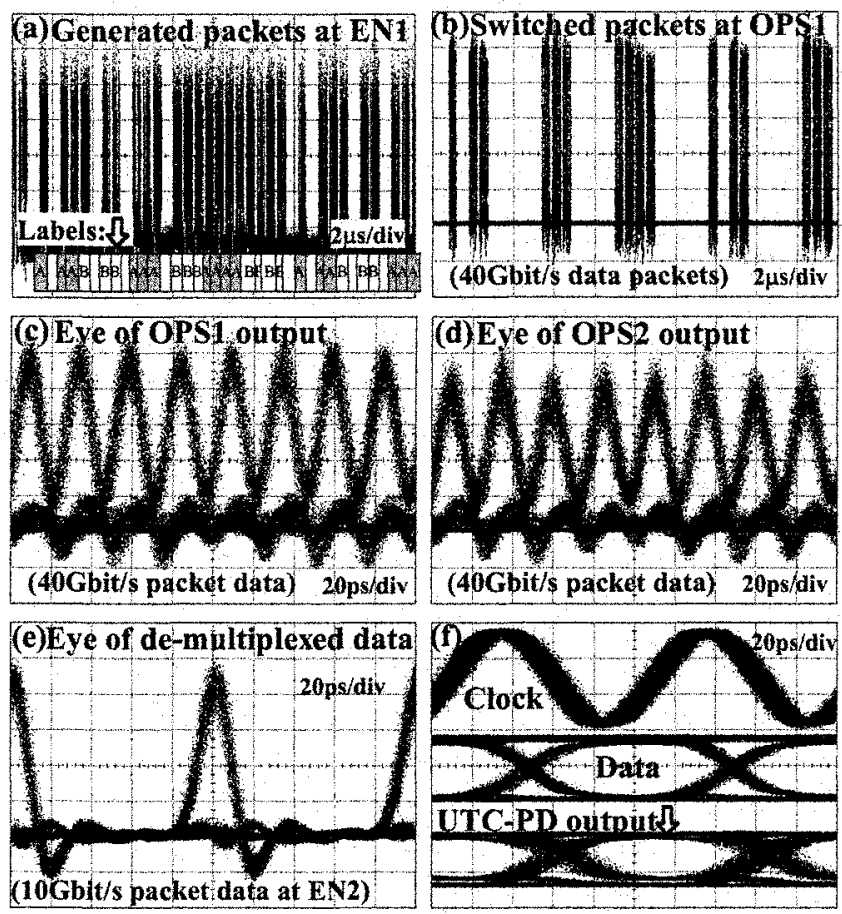

Figure 7. Measured waveforms of experimental demonstration II 
Figure 7 represents measured waveforms. Figures 7 (a) to (e) are generated 40Gbit/s packets with two different labels at EN1, switched $40 \mathrm{Gbit} / \mathrm{s}$ packets at OPS 1 , eye diagram of OPS 1 output, eye of switched $40 \mathrm{Gbit} / \mathrm{s}$ packets at OPS 2 , and eye of de-multiplexed 10Gbit/s packet data at EN2, respectively. Figure 7(f) shows UTC-PD output, recovered clock, and recovered data. These results show good performance of optical code label processing based optical packet switching and our proposed OPS receiver.

\section{CONCLUSIONS}

A novel preamble free optical packet receiver has been proposed. 10Gbit/s optical packets streams with preamble-less payload data from 1000 to 4000 bitslong and arbitrary intervals from nanosecond to microseconds have been generated. The packets stream has been asynchronously received by optical packet $3 R$ receiver. Instantaneous clock and payload data recovery in less than a bit period $(<100 \mathrm{ps})$ have been experimentally demonstrated. Clock and payload data recovery have been tested with various type of optical packets streams. This $3 \mathrm{R}$ receiver can be used to optical burst switched (OBS) networks as well as OPS networks. Preamble-less optical packet clock and data recovery with two hop 40Gbit/s optical packet switching based on all-optical code label processing has been also experimentally demonstrated.

\section{ACKNOWLEDGMENTS}

The authors would like to thank T. Hanyu, H. Sumimoto, T. Makino, Y. Tomiyama, and Y. Awaji of NICT for their collaboration in experiment. The authors would also like to thank Y. Matsyshima and M. Nagao of NICT for their encouragement.

\section{REFERENCES}

[1] D.J. Blumenthal et al, IEEE Photon. Technol. Lett., vol. 11, no., pp. 1497-1499, 1999.

[2] K. Kitayama et al, IEEE Photon. Technol. Lett., vol.11, no.12, pp.1689-1691, 1999.

[3] S.B. Yoo, OFC2003, vol. 2, no. FS5, pp. 797-798, 2003.

[4] N. Wada et al, OFC2003, vol. 2, no. FS7, pp. 801-802, 2003.

[5] N. Calabretta et al, ECOC2003, vol. 2, no. Tu1.4.5, pp. 178-179, 2003.

[6] K. Shrikhande et al, OFC2001, no. ThG2, 2001.

[7] M. Duelk et al, OFC2003, no. PD8, 2003.

[8] S. Kimura et al, ECOC2003, vol. 4, no. Th3.3.3, pp. 1042-1043, 2003. 